\title{
Anabases
}

ANABASES Traditions et réceptions de l'Antiquité

$7 \mid 2008$

Varia

\section{Le Miroir des classiques}

\section{Frédéric Duval}

\section{(2) OpenEdition}

Journals

Édition électronique

URL : http://journals.openedition.org/anabases/2545

DOI : 10.4000/anabases. 2545

ISSN : 2256-9421

\section{Éditeur}

E.R.A.S.M.E.

\section{Édition imprimée}

Date de publication : 1 mars 2008

Pagination : 266-271

ISSN : 1774-4296

\section{Référence électronique}

Frédéric Duval, «Le Miroir des classiques », Anabases [En ligne], 7 | 2008, mis en ligne le 01 décembre 2011, consulté le 14 novembre 2019. URL : http://journals.openedition.org/anabases/2545 ; DOI : 10.4000/anabases.2545

Ce document a été généré automatiquement le 14 novembre 2019.

(c) Anabases 


\title{
Le Miroir des classiques
}

\author{
Frédéric Duval
}

1 Catalogue informatisé des traductions médiévales en français et en occitan des auteurs classiques, le Miroir des classiques a commencé à paraître sur le site de l'École des chartes, en mars 2007, dans la collection de publications électroniques Elec ${ }^{1}$. Il est rédigé par Frédéric Duval et Françoise Vielliard. Sont présentées, sous le titre de chaque ouvrage traduit, les différentes traductions dans leur ordre chronologique ainsi que leurs remaniements éventuels. Pour chaque traduction, caractérisée rapidement, est fournie une analyse précise des manuscrits et des éditions (incunables et éditions du $\mathrm{XVI}^{\mathrm{e}}$ siècle) qui la conservent. On trouvera dans les lignes qui suivent une présentation sommaire du projet, résumant l'introduction plus détaillée consultable sur le site ${ }^{2}$.

\section{Genèse du projet}

2 L'intérêt pour les traductions médiévales des classiques s'est éveillé dans le cours du $\mathrm{XIX}^{\mathrm{e}}$ siècle ${ }^{3}$ chez des érudits, souvent chartistes, qui y sont venus par l'étude des manuscrits. On rappellera que la synthèse fondamentale de Paul Meyer ${ }^{4}$ sur les premières compilations d'histoire ancienne a comme point de départ l'analyse détaillée du manuscrit fr. 23083 de la Bibliothèque nationale de France pour les Faits des Romains et du manuscrit fr. 246 pour l'Histoire ancienne jusqu'à César et qu'on lui doit aussi le premier essai de recensement des traductions de Végèce ${ }^{5}$. Enfin, nombre des notices de manuscrits qu'il a établies, publiées dans Romania, dans le Bulletin de la Société des anciens textes français ou dans les Notices et extraits des manuscrits des bibliothèques publiques de France constituent, encore aujourd'hui, quelquefois l'unique documentation disponible sur les textes qu'ils contiennent.

3 De même c'est l'étude du manuscrit 590 de Chantilly qui a fourni à Léopold Delisle la matière d'une synthèse sur Jean d'Antioche, traducteur de la Rhétorique de Cicéron ${ }^{6}$. On doit aussi à Delisle le premier inventaire des traductions de la Consolation de Boèce ${ }^{7}$ et on n'oubliera pas que ses Recherches sur la librairie de Charles $V$ fondent encore aujourd'hui les études d'ensemble sur les traducteurs de Charles $\mathrm{V}^{8}$. Antoine Thomas ${ }^{9}$, ainsi que Robert Bossuat ${ }^{10}$, sont représentatifs de cette tradition chartiste d'étude des 
traductions, dans laquelle s'inscrivent aussi nombre de thèses d'École des chartes dont une partie est restée inédite.

4 Jacques Monfrin s'inscrivit dans cette voie en présentant en 1955 à l'Académie des inscriptions et belles-lettres un Inventaire critique des traductions d'auteurs anciens $d u$ XIII au $\mathrm{XV}^{e}$ siècle. Alors que l'Inventaire restait inédit, Jacques Monfrin a continué toute sa vie de travailler les dossiers ouverts lors de son élaboration, comme en témoignent ses séminaires de l'École pratique des Hautes Études, plusieurs de ses articles et les thèses d'École des chartes qu'il a dirigées. C'est de cet inventaire qu'est née l'idée de confectionner le Miroir des classiques.

5 Il existe, certes, plusieurs répertoires sommaires que le chercheur peut consulter pour repérer les traductions médiévales d'auteurs classiques, mais leur contenu est souvent très succinct : en 1954, R.R. Bolgar ${ }^{11}$ a publié un tableau des différentes traductions des auteurs classiques en anglais, français, allemand, italien, espagnol et antérieures à 1600, mais il est surtout utile à partir de l'apparition du livre imprimé ; en 1970, Robert H. Lucas ${ }^{12}$ a fait paraitre une liste d'auteurs classiques traduits au Moyen Âge, mais sa documentation, généralement incomplète, révèle d'assez nombreuses confusions; le Dictionnaire des traducteurs et de la littérature traduite publié par Paul Chavy en $1986{ }^{13}$ inclut bien sûr les traductions des classiques, mais vu le nombre de traductions envisagées, les informations données sur notre corpus ne peuvent être que sommaires.

\section{Définition du corpus}

Le Miroir des classiques a l'ambition de recenser les traductions des «classiques » faites en français, continental ou insulaire, en occitan et en francoprovençal au Moyen Âge. Il est donc important, s'agissant de traductions, de définir le corpus des textes sources et celui des textes cibles.

Corpus des textes sources

Si l'on admet que les auteurs de l'Antiquité gréco-romaine forment une partie bien individualisée au sein d'un corpus plus vaste d'auctoritates et que la notion de «classique » est opératoire pour le Moyen Âge ${ }^{14}$, force est d'établir une limite chronologique. 529 est la date de fermeture de l'Académie de Platon par Justinien et celle de la fondation de la communauté du Mont-Cassin par saint Benoît. Boèce, qui avait l'intention de traduire les œuvres complètes de Platon et d'Aristote pour les rendre accessibles à l'Occident latin, est mort en 524. Ces dates (524-529) marquent symboliquement la fin d'une tradition et le début d'une autre. Émerge alors, avec la conscience du passage à des temps nouveaux, la nécessité d'une synthèse des connaissances: l'élaboration du Corpus juris civilis (528-534) peut être interprétée comme le parallèle juridique du projet non abouti de synthèse philosophique par Boèce. Sont donc définis dans le Miroir des classiques comme « classiques » les auteurs et les textes païens antérieurs à la seconde moitié $\mathrm{du} \mathrm{vl}^{\mathrm{e}}$ siècle.

$8 \quad$ Le point de vue du Miroir des classiques étant celui du Moyen Âge, les textes apocryphes y sont inclus systématiquement, alors que Jacques Monfrin les avait exclus, même s'ils sont quelquefois postérieurs au $\mathrm{VI}^{\mathrm{e}}$ siècle et explicitement chrétiens. Le De vetula que la critique moderne attribue à Richard de Fournival (xIII ${ }^{\mathrm{e}}$ siècle) est un poème en trois chants dans lequel Ovide est censé raconter un épisode de sa vie amoureuse : il a été attribué par le Moyen Âge à Ovide et comme tel a été traduit en français par Jean Le 
Fèvre en 1376. De même le traité de morale du moine Martin de Braga (510-519) intitulé Formula honestae vitae est attribué par le Moyen Âge à Sénèque et a été traduit trois fois $\mathrm{du} \mathrm{XIII}^{\mathrm{e}} \mathrm{au} \mathrm{XV}^{\mathrm{e}}$ siècle. Si nous avons retenu les apocryphes, nous avons en revanche éliminé les textes qui, entre le $\mathrm{x}^{\mathrm{e}}$ et le $\mathrm{XII}^{\mathrm{e}}$ siècles, ont cherché à remplacer les classiques jugés dangereux pour l'orthodoxie de la foi.

Corpus des textes cibles

Il est quelquefois difficile de trancher entre traductions, adaptations et remaniements. Deux relations ont été prises en compte : d'une part le rapport qu'entretient le texte antique avec sa ou ses traductions vernaculaires; d'autre part le rapport entre une traduction vernaculaire avec ses remaniements éventuels.

10 Sur le rapport entre texte antique et traduction, le Miroir des classiques adopte une définition restrictive de la traduction. Ne sont en effet retenues dans un premier temps comme traductions que celles qui sont présentées comme telles, qui respectent un certain degré de littéralité et qui sont d'ailleurs souvent précédées d'un prologue qui justifie la démarche du traducteur.

11 Sur le rapport entre une traduction vernaculaire avec ses remaniements éventuels, toutes les péripéties textuelles doivent être considérées, même si elles éloignent un peu plus du texte source : ainsi la traduction des décades I, III et IV de Tite-Live faite par Pierre Bersuire à la demande de Jean le Bon a été retouchée au début du Xve siècle ; plus tard, le bourguignon Jean Mansel l'a intégrée en grande partie dans la compilation historique intitulée la Fleur des histoires qui a connu elle-même une version brève et une version longue. L'ensemble du dossier figure dans la notice consacrée à Tite-Live.

Le terminus ad quem du corpus des traductions est 1500. Cette coupure est arbitraire du point de vue de l'activité traductrice, puisqu'un nouveau mouvement de traduction s'est amorcé dans les dernières décennies $d u \mathrm{xv}^{\mathrm{e}}$ siècle, où l'on commence à traduire des œuvres moins didactiques : une partie du théâtre de Térence passe à deux reprises en français dans la seconde moitié du siècle; Octovien de Saint-Gelais traduit les Héroïdes d'Ovide en 1496 et l'Énéide de Virgile en 1500. La date de 1500 est une coupure commode - Jacques Monfrin l'avait déjà souligné - qui permet d'exclure les très nombreuses traductions réalisées dans les premières années $\mathrm{du} \mathrm{xvI}^{\mathrm{e}}$ siècle, alors que le contexte, tant social qu'intellectuel, de production des traductions, se modifie sensiblement.

13 Le corpus des témoins du texte cible comporte les manuscrits subsistants et les éditions anciennes. Le terminus ad quem a été fixé pour les témoins à la fin $\mathrm{du} \mathrm{xvI}^{\mathrm{e}}$ siècle: il permet de prendre en compte le passage du manuscrit à l'imprimé et d'éclairer les rapports entre l'appropriation vernaculaire des classiques et la «Renaissance » française $d u x{ }^{e}$ siècle. De même sont signalés, dans la mesure du possible, les possesseurs des manuscrits jusqu'à la fin du Xvi ${ }^{\mathrm{e}}$ siècle.

\section{Présentation des notices}

14 Les notices comportent trois niveaux: le texte source, la/les traduction(s), le(s) témoin(s). À chaque niveau correspond une bibliographie réduite à l'essentiel.

15 Le texte source

À ce premier niveau sont données de brèves indications sur la fortune latine du texte puis sur sa réception française ailleurs que dans les traductions traitées dans la notice. 
On y présente rapidement les textes français qui n'ont pas été retenus bien qu'ils entretiennent un rapport de dépendance ou de référence avec le texte latin qui fait l'objet de la notice.

Dans une étape ultérieure, le Miroir intégrera des notices limitées à ce premier niveau : il est en effet important de rendre compte de la réception vernaculaire d'auteurs classiques qui n'ont pas suscité de traductions au sens strict, mais qui ont fait l'objet d'adaptations ou de traductions partielles insérées dans des compilations. Des historiens comme Salluste, Suétone ou Lucain, des poètes comme Horace, Juvénal ou Virgile, par exemple, doivent y avoir leur place.

\section{La traduction}

Classées par ordre chronologique, les traductions portent le titre que leur a conféré la critique, à défaut celui qui est le plus fréquemment donné par les manuscrits. Suit une courte notice, reposant sur les acquis de la recherche, qui précise la date de traduction, les procédés de traduction les plus marquants, les liens possibles avec d'autres traductions, l'ampleur, la nature et les sources des développements imputés au traducteur.

Dans plusieurs notices sont édités des extraits, surtout quand aucune édition n'en est disponible : il s'agit généralement du prologue dont on sait l'importance pour l'étude des traductions médiévales. Figurent ensuite les incipit et explicit d'après l'édition critique faisant référence et à défaut d'après un manuscrit dont la critique a signalé la qualité des leçons. Lorsque la traduction a été remaniée, le remaniement est présenté à sa suite selon les mêmes critères.

Les témoins

Il s'agit de la partie la plus longue de la notice: sur ce point le Miroir des classiques diverge de l'Inventaire de Jacques Monfrin qui donnait une liste de manuscrits limitée à l'indication de la cote, de la date et de la provenance.

Les notices de manuscrits suivent le schéma suivant :

- nombre de feuillets et support ;

- datation: elle se compose d'une date de lieu, peu systématique, et d'une date de temps ;

- contenu : le titre de chaque texte copié dans le manuscrit est donné, sauf si ce dernier en compte plus de dix ;

- copie : la présence d'une mention de copiste comporte un renvoi au feuillet où elle apparaît et elle est quelquefois reproduite ;

- illustration : sont précisées les attributions données par la bibliographie, le nombre, la taille et la localisation des miniatures. Lorsque le nombre des miniatures est supérieur à dix, on se contente des deux premiers éléments ;

- possesseurs : les mentions de possession du Moyen Âge et $d u x I^{e} s$. sont toujours signalées, parfois reproduites ;

- sigle : le ou les sigles attribué(s) aux manuscrits par les éditeurs et philologues sont donnés en fin de notice ;

- bibliographie.

21 Les notices d'imprimés donnent, dans l'ordre, le titre, le lieu d'édition, l'imprimeurlibraire, la date, le format, l'indication de la présence d'une illustration et le nombre de feuillets, quand l'édition est foliotée ou que le compte des feuillets a déjà été fait. La transcription du titre ne respecte ni les majuscules ni la mise en page de l'imprimé ancien, mais le titre est chaque fois donné in extenso. Le contenu de l'imprimé est 
détaillé suivant les principes de présentation qui ont été adoptés pour les manuscrits. L'exemplaire qui a été consulté est identifié par sa cote. La bibliographie renvoie à la description la plus récente ou la plus complète sans citer l'ensemble des répertoires usuels.

Au 20 octobre 2007, le Miroir présente 47 traductions médiévales de classiques latins. Les auteurs grecs seront traités dans un second temps. Les notices, publiées au fur et à mesure de leur achèvement, devraient prochainement voir leur nombre s'accroître.

\section{NOTES}

1. L'adresse du Miroir des classiques est la suivante : http://elec.enc.sorbonne.fr/miroir/.

2. On pourra également se reporter à F. DUVAL et F. VIELLIARD, "La transmission des auteurs classiques dans les traductions en français et en occitan du XIII ${ }^{\mathrm{e}}$ au XV siècle", in P. NOBEL (éd.), La transmission des savoirs au Moyen Âge et à la Renaissance, t. I : du XII $e^{e} a u X^{e}$ siècle, Besançon, 2005, p. 363-384.

3. On trouvera un historique des études sur les traductions médiévales remontant au XVIII ${ }^{\mathrm{e}}$ siècle sous la plume de F. BÉRIER, in La littérature française aux XIV et $X V^{e}$ siècles, t. 8, vol. I (partie historique) du Grundriss der romanischen Literaturen des Mittelalters, Heidelberg, 1988, p. 219-265 "La traduction en français", part. Historique des études, p. 219-220.

4. P. MEYER, "Les premières compilations françaises d'histoire ancienne", Romania 14 (1885), p. 1-85.

5. P. MEYER, "Les anciens traducteurs français de Végèce et en particulier Jean de Vignay", Romania 25 (1896), p. 401-408.

6. L. DELISLE, "Notice sur la rhétorique de Cicéron, traduite par Maître Jean d'Antioche, ms. 590 du Musée Condé", Notices et extraits des manuscrits des bibliothèques publiques de France 36 (1899), p. 1-63 ; ID., "Maître Jean d'Antioche, traducteur et frère Guillaume de Saint-Étienne, hospitalier", in Histoire littéraire de la France, t. 33, 1906, p. 1-40.

7. L. DELISLE, “Anciennes traductions françaises de la Consolation de Boèce conservées à la Bibliothèque nationale", Bibliothèque de l'École des chartes 34 (1873), p. 5-32.

8. L. DELISLE, Recherches sur la librairie de Charles V, 2 vol., Paris, 1905, en part., t. I, p. 82-119.

9. A. тномаs, "Anonyme italien, auteur d'une traduction française des Lettres de Sénèque à Lucilius", in Histoire littéraire de la France, t. 35, 1921, p. 633-635 ; ID., "Traductions françaises de la Consolatio philosophice de Boèce", in Histoire littéraire de la France, t. 37, 1938, p. 419-470 ; L. ROYER et A. THомAs, La somme du Code, texte dauphinois de la région de Grenoble, publié d'après un manuscrit du XIII ${ }^{\mathrm{e}}$ siècle appartenant à la Bibliothèque du château d'Uriage, Paris, 1929.

10. R. Bossuat, “Anciennes traductions françaises du De officiis de Cicéron”, Bibliothèque de l'École des chartes 96 (1935), p. 246-284 ; “Jean Miélot, traducteur de Cicéron”, Bibliothèque de l'École des chartes 99 (1938), p. 82-124 ; "Traductions françaises des Commentaires de César à la fin du Xve siècle", Bibliothèque d'Humanisme et Renaissance 3 (1943), p. 253-411; "Vasque de Lucène, traducteur de Quinte-Curce (1468)”, Bibliothèque d'Humanisme et Renaissance 8 (1946), p. 197-246 ; "Jean de Rouvroy, traducteur des Stratagèmes de Frontin", Bibliothèque d'Humanisme et Renaissance 22 (1960), p. 273-286 et 469-489 ; “Raoul de Presles”, dans Histoire littéraire de la France, t. 40, 1974, p. $113-186$. 
11. R. R. BOLGAR, "The translation of the greek and roman classical authors before 1600", in The Classical Heritage and its Beneficiaries, Cambridge, 1954, p. 506-541.

12. R. H. LUCAS, "Mediaeval French Translations of the Latin Classics to 1500", Speculum 45 (1970), p. 225-253.

13. P. CHAVY, Traducteurs d'autrefois. Moyen Âge et Renaissance. Dictionnaire des traducteurs et de la littérature traduite en ancien et en moyen français (842-1600), 2 vol. Paris, 1986.

14. Sur ce point controversé, voir l'introduction du Miroir sur le site, mais aussi J. LEEKER, "Formes médiévales de la vénération de l'Antiquité", in P. NOBEL (éd.), La transmission des savoirs au Moyen Âge et à la Renaissance, Besançon, 2005, t. I, p. 77-98 et F. DUVAL, "Petite enquête lexicologique sur l'Antiquité", in L'Antiquité entre Moyen Âge et Renaissance. L'Antiquité dans les livres produits au Nord des Alpes entre 1350 et 1520 (Actes du colloque de Paris, 8-10 mars 2006), à paraître.

\section{AUTEUR}

FRÉDÉRIC DUVAL

fduval@atilf.fr 